\title{
Reply to Lasersohn, MacFarlane, and Richard
}

\author{
Herman Cappelen · John Hawthorne
}

Published online: 20 November 2010

(C) The Author(s) 2010. This article is published with open access at Springerlink.com

\section{Précis of Relativism and Monadic Truth}

The beginning of the 21 st century saw something of a comeback for relativism within analytical philosophy. Relativism and Monadic Truth has three main goals. First, we wished to clarify what we take to be the key moving parts in the intellectual machinations of self-described relativists. Second, we aimed to expose fundamental flaws in those argumentative strategies that drive the pro-relativist movement and precursors from which they draw inspiration. Third, we hoped that our polemic would serve as an indirect defence of a traditional and natural picture concerning truth. According to this picture, what we call 'Simplicity', the fundamental structure of semantic reality is best revealed by construing truth as a simple monadic property of propositions that serve as the objects of belief, assertion, meaning and agreement.

Our project was not a straightforward one. So-called relativists are not uniform in their key ideology, are often sloppy, casual, obscure or confused in their selfcharacterization, and differ in their argumentative emphasis among themselves and over time, thereby presenting a target that is both amorphous and shifty. This is an area where parties will frequently claim not to understand each other and where certain parties will sometimes accuse others of failing to make any sense at all. In such a situation any effort to impose order will inevitably strike some parties as tendentious and unfair. That said, we felt that we had enough of a grip on the relativist movement to recognize it as a degenerating research program, and enough of a grip on the resources available to Simplicity to see it as largely unscathed.

H. Cappelen ( $ه$ )

CSMN, IFIKK, Postboks 1020, Blindern, 0315 Oslo, Norway

e-mail: herman.cappelen@gmail.com

J. Hawthorne

Magdalen College, Oxford University, OX1 4AU, Oxford, UK

e-mail: john.hawthorne@philosophy.ox.ac.uk 
Relativism is often depicted as continuous with a larger tradition in semantics that parameterizes the meanings of utterances and the objects of belief. By the lights of such parameterizations, the relevant contents are not true or false simpliciter but instead true or false relative to this or that parameter value. Such parameterizations are encouraged by talk of propositions being true relative to worlds and times. But insofar as these latter ways of talking are accompanied by a rejection of the claim that propositions are straightforwardly true or false, they already constitute a misstep. The ideology of truth at a world or time is best seen as part of a theoretical vocabulary that can sit alongside, and which is indeed less fundamental than, the ideology of monadic truth and falsehood. One sometimes hears relativism presented in a way that makes many of its core commitments seem somewhat innocent: 'We have already learned that truth is relative to worlds and times. There is nothing very drastic about adding a few more parameters'. Our reply is that we have learned no such thing. These ways of talking should never have been seen as challenges to monadic truth or falsity, and so cannot pave the way for relativism.

As we see things, there are three main ideas that drive relativism-notwithstanding the dizzying array of articulations that we find in the literature.

- First, relativists proliferate parameters. They draw inspiration from those who reject simple truth and falsity in favour of parameterization of truth to worlds and times and then add a range of further putative parameters. (Those that replace simple truth or falsity with truth relative to times and worlds, and hence insist that the content of a given assertion is neutral concerning times and worlds still concede that particular times and worlds might be relevant to the felicity of a particular assertion. Similarly, for these extra parameters.)

- Second, relativists add a disquotational truth predicate to their expressive resources. Roughly, just as ' $S$ ' will be true relative to certain parameter values and false relative to others, so " $\mathrm{S}$ ' is true" will be parameterized in exactly the same way.

- Third, they embrace non-parameterization of belief and meaning ascriptions. Roughly speaking, various parameters for a sentence become redundant when that sentence is embedded in a meaning or belief report. Thus, for example, while 'Backpacks are cool' may be true or false relative to a standard of taste 'Certain philosophers believe that backpacks are cool' will not exhibit such relativity.

In Chap. 1 we showed how, with these pieces in place, we can illuminate various relativist ideas including 'no fault disagreement' and 'assessment sensitivity'.

Chapter 2 is devoted to a discussion of various diagnostics for shared content. Assumptions about content sharing are central to relativism and its precursors. For example, relativists about taste, often assume that what I believe when I accept 'Kebabs are tasty' is what someone disbelieves then they reject 'Kebabs are tasty'. By way of ground clearing we thought it helpful to evaluate various diagnostics for shared content. Contrary to what many implicitly or explicitly assume, collective 'say that' and 'believe that' claims are far inferior to collective 'agree that' data. Disagreement data, we argue, has even more evidential force.

Chapter 3 looks at a style of pro-parameterization argument made prominent in Kaplan. Related ideas are promoted by Lewis and are frequently paraded in current 
relativist literature. We found that the argument was inexplicit and underdeveloped in various spots. We tried to be explicit about the relevant assumptions. We found all of them to be problematic in certain of their applications and were sceptical about whether any compelling operator arguments were left standing.

Chapter 4 is devoted to a case study: predicates of personal taste. This is one of the best cases for relativism, in part because the various shared content diagnostics tell prima facie in its favour. Our discussion combines a number of themes. Relativists typically fail to pay attention to crucial linguistic details, neglecting noise introduced by generic constructions, neglecting important distinctions among and between various uses of 'fun', 'disgusting' and other predicates of taste, neglecting cases that are very problematic for the view, notably ones where superficial contradiction is not accompanied by a judgment of disagreement. Meanwhile, relativists typically argue against a contextualist strawman, failing to pay attention to the array of resources available to sophisticated contextualists. Further, relativists typically fail to notice that ordinary people are often inclined to a naïve realism and that disagreement verdicts seem to wax and wane precisely to the extent that such naivety gains and loses its hold. As well as outlining and defending a sophisticated contextualism, we outline a battery of arguments against relativism. One trouble spot worth noting is that the relativist will have far more difficulty than is usually recognized in distinguishing her own position from that of a naïve chauvinistic realist.

Personal taste is one of the favoured hunting grounds for relativism. Even here, the advantages of relativism prove largely chimeral. The pattern of breakdown is likely to be replicated in other areas-epistemic modals, conditionals, future contingents, and so on-where relativism has been promoted as a viable option. We expect to see many more relativist proto-theories advanced in the near future. We doubt that very much will come of them.

Open Access This article is distributed under the terms of the Creative Commons Attribution Noncommercial License which permits any noncommercial use, distribution, and reproduction in any medium, provided the original author(s) and source are credited. 